\title{
Particle propagation in cosmological backgrounds
}

\author{
Daniel Arteaga \\ Departament de Física Fonamental. Facultat de Física. Av. Diagonal 647, 08028 \\ Barcelona (Spain) \\ E-mail: darteaga@ub.edu
}

\begin{abstract}
We study the quantum propagation of particles in cosmological backgrounds, by considering a doublet of massive scalar fields propagating in an expanding universe, possibly filled with radiation. We focus on the dissipative effects related to the expansion rate. At first order, we recover the expected result that the decay rate is determined by the local temperature. Beyond linear order, the decay rate has an additional contribution governed by the expansion parameter. This latter contribution is present even for stable particles in the vacuum. Finally, we analyze the long time behaviour of the propagator and briefly discuss applications to the transPlanckian question.
\end{abstract}

\section{Introduction}

In this contribution we study the quantum propagation of particles in a cosmological background. We are particularly interested in understanding the dissipative phenomena related to the time dependence of the metric. To this end, we analyze the propagator of a massive particle which interacts with a massless radiation field in an expanding universe. Several points must be considered.

First, we are dealing with an interacting field theory in a curved spacetime. In this situation, the asymptotic in and out vacua generally do not coincide. Being interested in expectation values, rather than in in-out matrix elements, we adopt the Keldysh-Schwinger formalism, or Closed Time Path (CTP) method [1-3] in curved spacetime [4-7].

Second, as it is well known, in a curved spacetime there is no single definition for the vacuum nor for the concept of particle. We face this issue by working within the adiabatic approximation [8]: the massive particles will have their Compton wavelengths much smaller than the typical curvature radius of the universe (in our case, the Hubble radius).

Third, as explained in [9], in theories such as QED or perturbative quantum gravity, dissipative effects appear only at two loops, because the one-loop diagrams which could lead to dissipation vanish on the mass shell. Here, in order to keep the calculations simple, we have chosen a simple, yet physically meaningful, model which exhibits 
dissipation at one loop. We expect the behaviour of QED or perturbative quantum gravity to be similar at two loops.

In this contribution we compute the retarded self-energy of the lightest field in a massive doublet which propagates in a thermal bath of massless particles in an expanding universe, and from it we extract the decay rate. Notice that in the Minkowski vacuum the excitations of the lighter field are stable, hence the decay rate is zero. We concentrate on the physical insights and summarize the main results. A more detailed account will be given in separate publications $[10,11]$

The contribution is organized as follows. In section 2 we introduce the model and motivate the use of the adiabatic approximation for the massive fields. In section 3 we present the results for the imaginary part of the self-energy and the decay rate. In section 4 we study the time evolution of the interacting propagator. Finally, in section 5 we summarize the main points of the contribution and discuss its relevance to the trans-Planckian question. We use a system of natural units with $\hbar=c=1$, and the metric has the signature $(-,+,+,+)$.

\section{The model}

We consider spatially isotropic and homogeneous Friedmann-Lemaître-RobertsonWalker models with flat spatial sections:

$$
\mathrm{d} s^{2}=-\mathrm{d} t^{2}+a^{2}(t) \mathrm{d} \mathbf{x}^{2} .
$$

The particle model is the following: two massive fields $\phi_{m}$, and $\phi_{M}$, interacting with a massless field, $\chi$, via a trilinear coupling. The total action is $S=S_{m}+S_{M}+S_{\chi}+S_{\text {int }}$, where each term is given by

$$
\begin{aligned}
& S_{m}=\frac{1}{2} \int \mathrm{d} t \mathrm{~d}^{3} \mathbf{x} a^{3}(t)\left(\left(\partial_{t} \phi_{m}\right)^{2}-\frac{1}{a^{2}(t)}\left(\partial_{\mathbf{x}} \phi_{m}\right)^{2}-m^{2} \phi_{m}^{2}\right), \\
& S_{M}=\frac{1}{2} \int \mathrm{d} t \mathrm{~d}^{3} \mathbf{x} a^{3}(t)\left(\left(\partial_{t} \phi_{M}\right)^{2}-\frac{1}{a^{2}(t)}\left(\partial_{\mathbf{x}} \phi_{M}\right)^{2}-M^{2} \phi_{M}^{2}\right), \\
& S_{\chi}=\frac{1}{2} \int \mathrm{d} t \mathrm{~d}^{3} \mathbf{x} a^{3}(t)\left(\left(\partial_{t} \chi\right)^{2}-\frac{1}{a^{2}(t)}\left(\partial_{\mathbf{x}} \chi\right)^{2}-\xi R(t) \chi^{2}\right), \\
& S_{\text {int }}=g M \int \mathrm{d} t \mathrm{~d}^{3} \mathbf{x} a^{3}(t) \phi_{m} \phi_{M} \chi
\end{aligned}
$$

with $R(t)$ being the Ricci scalar. We assume that the massless field is conformally coupled to gravity, so that $\xi=1 / 6$. It is useful to work with rescaled massive fields defined by $\bar{\phi}(t, \mathbf{x}):=[-g(t, \mathbf{x})]^{1 / 4} \phi(t, \mathbf{x})=a^{3 / 2}(t) \phi(t, \mathbf{x})$.

We consider the two massive fields having large masses but with a small mass difference $\Delta m:=M-m \ll M$. As shown in [12], the model can be interpreted as a field-theory description of a relativistic two-level atom (of mass $m$ and energy gap $\Delta m$ ) interacting with a scalar radiation field $\chi$. The radiation field $\chi$ is assumed to be at some conformal temperature $\theta$ (which can eventually be zero). The corresponding physical temperature, as well as the Hubble rate $H(t):=\dot{a}(t) / a(t)$, are chosen to be 
much smaller than the masses of the fields. These restrictions ensure that the number of massive particles is strictly conserved. The non-trivial dynamics concerns the transitions between the two massive fields accompanied by emission and absorption of massless quanta.

In a curved spacetimes it is not a trivial task to compute even the free field vacuum propagators. For massless conformally coupled fields there is a natural vacuum state, the conformal vacuum. Propagators in this vacuum, when expressed in conformal time, essentially correspond to the flat spacetime propagators. For the massive fields, rather than attempting to find the exact free propagator, we will exploit the fact that their Compton wavelengths is much smaller than the Hubble length $H^{-1}$. In this regime, the adiabatic (WKB) approximation is valid and explicit expressions for the free propagators can be computed $[8,10]$ — see for instance (19).

\section{The self-energy and decay rates}

In this section we consider the interacting retarded Green function

$$
G_{\mathrm{R}}\left(t, t^{\prime} ; \mathbf{p}\right):=\theta\left(t-t^{\prime}\right)\left\langle\left[\hat{\phi}_{m \mathbf{p}}(t), \hat{\phi}_{m \mathbf{p}}\left(t^{\prime}\right)\right]\right\rangle
$$

within the adiabatic approximation. It is related to the retarded self-energy $\Sigma_{\mathrm{R}}$ via [12]

$$
\begin{aligned}
G_{\mathrm{R}}\left(t, t^{\prime} ; \mathbf{p}\right)= & G_{\mathrm{R}}^{(0)}\left(t, t^{\prime} ; \mathbf{p}\right) \\
& -i \int \mathrm{d} s \mathrm{~d} s^{\prime} \sqrt{-g(s)} \sqrt{-g\left(s^{\prime}\right)} G_{\mathrm{R}}^{(0)}(t, s ; \mathbf{p}) \Sigma_{\mathrm{R}}\left(s, s^{\prime} ; \mathbf{p}\right) G_{\mathrm{R}}\left(s^{\prime}, t^{\prime} ; \mathbf{p}\right)
\end{aligned}
$$

where $G_{\mathrm{R}}^{(0)}\left(t, t^{\prime} ; \mathbf{p}\right)$ is the free retarded propagator. In terms of the rescaled fields, $\bar{\phi}(t ; \mathbf{p})=a^{3 / 2} \phi(t ; \mathbf{p})$, the above relation becomes

$\bar{G}_{\mathrm{R}}\left(t, t^{\prime} ; \mathbf{p}\right)=\bar{G}_{\mathrm{R}}^{(0)}\left(t, t^{\prime} ; \mathbf{p}\right)-i \int \mathrm{d} s \mathrm{~d} s^{\prime} \bar{G}_{\mathrm{R}}^{(0)}(t, s ; \mathbf{p}) \bar{\Sigma}_{\mathrm{R}}\left(s, s^{\prime} ; \mathbf{p}\right) \bar{G}_{\mathrm{R}}\left(s^{\prime}, t^{\prime} ; \mathbf{p}\right)$.

We assume that the massive fields are in the adiabatic vacuum, and that the massless field $\chi$ is in a thermal state, characterized by a fixed conformal temperature $\theta$. We will compute the imaginary part of the one-loop self energy to order $g^{2}$ in the adiabatic approximation, evaluated at the mass shell. It will be evaluated in a a frequency representation around the average time coordinate $T=\left(t_{1}+t_{2}\right) / 2$, by Fouriertransforming with respect to the difference coordinate $\Delta=t_{1}-t_{2}$, which amounts to a local frequency representation (it is further analyzed in next section). As for the spatial part, we work in the momentum representation to exploit conservation of the conformal momentum.

\subsection{Linear approximation to the scale factor}

As a first step, we approximate the evolution of the scale factor by a linear expansion:

$$
a(t) \approx a(T)[1+H(T)(t-T)] .
$$

This approximation for the scale factor is appropriate when considering physical temperatures which are much larger than the expansion rate (but still much smaller than 
the fields masses). On the mass shell, thermal corrections will dominate over curvature corrections, since the thermal energy scale is much larger than the curvature energy scale. Therefore, we expect the on-shell self-energy to be governed by the thermal bath at the instantaneous physical temperature at each moment of the expansion, $\theta / a(T)$.

The explicit calculation [10] confirms that the imaginary part of the on-shell selfenergy is given by that of a thermal bath in Minkowski at a physical temperature $\theta / a(T)$. In the limit in which the atoms are at rest this result is $[10,12]$

$$
\operatorname{Im} \bar{\Sigma}_{\mathbf{R}}(m, T ; \mathbf{0})=-\frac{g^{2}}{8 \pi} M \Delta m n_{\theta / a(T)}(\Delta m),
$$

where $n_{\theta / a(T)}(\Delta m)$ is the Bose-Einstein function:

$$
n_{\theta / a(T)}(\Delta m):=\frac{1}{e^{\Delta m a(T) / \theta}-1} .
$$

As in Minkowski spacetime, the self-energy corresponds to a decay rate,

$$
\Gamma=-\frac{1}{m} \operatorname{Im} \bar{\Sigma}_{\mathrm{R}}(m, T ; \mathbf{0})=\frac{g^{2}}{8 \pi} \Delta m n_{\theta / a(T)}(\Delta m),
$$

which amounts to the probability per unit time for the lightest state to absorb a massless particle from the thermal bath.

\subsection{Beyond linear order: vacuum effects}

When the expansion rate of the universe is of the order of the temperature or larger, vacuum effects become relevant. Energy conservation does not hold for energy scales of the order of the expansion rate, and therefore we expect new channels for the particle decay which will contribute to the imaginary part of the self-energy.

In order to study the vacuum effects we need to choose a explicit model for the evolution of the scale factor. For instance, in the case of de Sitter,

$$
a(t)=a(T) e^{H(t-T)},
$$

the vacuum contribution to the imaginary part of the retarded self-energy given by [11]

$$
\operatorname{Im} \bar{\Sigma}_{\mathrm{R}}(m, T ; \mathbf{0})=-\frac{g^{2}}{8 \pi} M \Delta m n_{H /(2 \pi)}(\Delta m),
$$

which coicides with the self-energy in a Minkowski thermal bath at a temperature $H /(2 \pi)$. The result is not unexpected since the effective de Sitter temperature [13] is recovered. The corresponding decay rate

$$
\Gamma=-\frac{1}{m} \operatorname{Im} \bar{\Sigma}_{\mathrm{R}}(m, T ; \mathbf{0})=\frac{g^{2}}{8 \pi} \Delta m n_{H /(2 \pi)}(\Delta m),
$$

amounts for the probability per unit time for the lightest field to emit a massless particle. Energy conservation forbids this process in Minkowski spacetime, but this restriction does not apply in an expanding universe. 


\section{Retarded propagator and self-energy in cosmology}

In expanding universes the propagators are no longer time-translation invariant. We can nevertheless always express the propagator in a frequency representation,

$$
\bar{G}_{\mathrm{R}}(\omega, T ; \mathbf{p}):=\int \mathrm{d} \Delta e^{i \omega \Delta} \bar{G}_{\mathrm{R}}(T+\Delta / 2, T-\Delta / 2 ; \mathbf{p}) .
$$

For short time differences as compared to the inverse expansion rate, i.e., $\left|t-t^{\prime}\right| \ll H^{-1}$, (5) can be diagonalized:

$$
\bar{G}_{\mathrm{R}}(\omega, T ; \mathbf{p})=\frac{-i}{\left[-i \bar{G}^{(0)}(\omega, T ; \mathbf{p})\right]^{-1}+\bar{\Sigma}_{\mathrm{R}}(\omega, T ; \mathbf{p})} .
$$

Fourier-transforming again we get the short-time behavior:

$$
\bar{G}_{\mathrm{R}}\left(t, t^{\prime} ; \mathbf{p}\right)=\frac{-i}{R_{\mathbf{p}}(T)} \sin \left[R_{\mathbf{p}}(T)\left(t-t^{\prime}\right)\right] e^{-\Gamma_{\mathbf{p}}(T)\left(t-t^{\prime}\right) / 2} \theta\left(t-t^{\prime}\right) .
$$

with

$R_{\mathbf{p}}^{2}(T):=E_{\mathbf{p}}^{2}(T)+\operatorname{Re} \bar{\Sigma}_{\mathrm{R}}\left(E_{\mathbf{p}}, T ; \mathbf{p}\right):=m^{2}+\frac{\mathbf{p}^{2}}{a^{2}(T)}+\operatorname{Re} \bar{\Sigma}_{\mathrm{R}}\left(E_{\mathbf{p}}, T ; \mathbf{p}\right)$

and

$$
\Gamma_{\mathbf{p}}(T):=-\frac{1}{R_{\mathbf{p}}(T)} \operatorname{Im} \bar{\Sigma}_{\mathrm{R}}\left(E_{\mathbf{p}}, T ; \mathbf{p}\right) .
$$

Therefore one recovers the usual interpretation, in which the real part of the self-energy corresponds to the energy shift, and in which the imaginary part corresponds to the decay rate. Notice that both quantities depend in general on time.

One may also be interested in considering large time lapses, and in this case the frequency representation of the propagator around the average time does not make sense. Lifting the short-time requirement, and only imposing the adiabatic approximation, the following expression for the evolution of the retarded propagator is found [10]:

$\bar{G}_{\mathrm{R}}\left(t_{1}, t_{2} ; \mathbf{p}\right)=\frac{-i}{\sqrt{R_{\mathbf{p}}\left(t_{1}\right) R_{\mathbf{p}}\left(t_{2}\right)}} \sin \left(\int_{t_{2}}^{t_{1}} \mathrm{~d} t^{\prime} R_{\mathbf{p}}\left(t^{\prime}\right)\right) e^{-\int_{t_{2}}^{t_{1}} \mathrm{~d} t^{\prime} \Gamma_{k}\left(t^{\prime}\right) / 2} \theta\left(t_{1}-t_{2}\right)$.

Notice that the long-time evolution of the propagator can be expressed in terms of integrals of quantities evaluated in the local frequency representation. Two time scales are clearely separated: the interaction timescale, in which the interaction process take place and in which the self-energy is evaluated, and the evolution timescale, which can be much longer and during which the propagators deviate significantly from the corresponding Minkowski expression. Equation (18) can be derived in a very similar way as the well-known adiabatic approximation for the free retarded propagator [8]:

$$
\bar{G}_{\mathrm{R}}^{(0)}\left(t_{1}, t_{2} ; \mathbf{p}\right)=\frac{-i}{\sqrt{E_{\mathbf{p}}\left(t_{1}\right) E_{\mathbf{p}}\left(t_{2}\right)}} \sin \left(\int_{t_{2}}^{t_{1}} \mathrm{~d} t^{\prime} E_{\mathbf{p}}\left(t^{\prime}\right)\right) \theta\left(t_{1}-t_{2}\right) .
$$




\section{Summary and discussion}

The goal of this contribution is to analyze the quantum effects in the propagation of interacting fields in a cosmological background. This issue may play an important role in justifying the non-trivial dispersion relations which have been used when addressing the trans-Planckian question in the context of black holes [14-17] and cosmology [18-21]. Interactions could indeed significantly modify the field propagation when approaching the event horizon of a black hole [22-25] or at primordial stages of inflation [9].

In our model, the masses of the fields were assumed to be much larger than the expansion rate of the universe. This was a key assumption, because it allowed to introduce the adiabatic (WKB) approximation, which not only makes the problem solvable, but also allows having a well-defined particle concept even in absence of asymptotic regimes. Within this approximation, the time-evolution of the interacting propagators can be computed from the integral of the retarded self-energy, evaluated on-shell in a frequency representation around the mid time.

The imaginary part of the self-energy determines the decay of the retarded propagator, and hence it is an expression of the dissipative properties. For temperatures higher than the expansion parameter the decay of the propagator is determined by the local temperature at each moment of expansion. For lower temperatures, the decay of the propagator is driven by the expansion rate of the universe. This second contribution, which is present even in the vacuum, can be interpreted as being a consequence of the absence of energy conservation at those energy scales comparable to the expansion rate.

The decay rate, derived from the imaginary part of the self-energy, has a secular character. Even small decay rates could thus give an important effect when integrated over large periods of time. The exact significance of the generically dissipative properties of the propagator will be further analyzed elsewhere [11].

\section{Acknowledgments}

I am very grateful with Renaud Parentani and Enric Verdaguer for a critical reading of the manuscript. This work is partially supported by the Research Projects MEC FPA2004-04582-C02-02 and DURSI 2005SGR-00082.

\section{References}

[1] J. S. Schwinger. J. Math. Phys., 2:407, 1961.

[2] L. V. Keldysh. Zh. Eksp. Teor. Fiz, 47:1515, 1965. [Sov. Phys. JEPT 20:1018, 1965].

[3] K.-C. Chou, Z.-B. Su, B.-L. Hao, and L. Yu. Phys. Rept., 118:1-131, 1985.

[4] R. D. Jordan. Phys. Rev. D, 33:444-454, 1986.

[5] E. Calzetta and B. L. Hu. Phys. Rev. D, 35:495-509, 1987.

[6] A. Campos and E. Verdaguer. Phys. Rev. D, 49:1861-1880, 1994.

[7] S. Weinberg. Phys. Rev. D, 72:043514, 2005.

[8] N. D. Birrell and P. C. W. Davies. Quantum fields in curved space. Cambridge University Press, Cambridge, England, 1982. 
[9] D. Arteaga, R. Parentani, and E. Verdaguer. Phys. Rev. D, 70:044019, 2004.

[10] D. Arteaga, R. Parentani, and E. Verdaguer. To appear in Int. J. Theor. Phys.

[11] D. Arteaga, R. Parentani, and E. Verdaguer. In preparation.

[12] D. Arteaga, R. Parentani, and E. Verdaguer. Int. J. Theor. Phys., 44:1665-1689, 2005.

[13] E. Mottola, Phys. Rev. D, 31:754-766, 1985.

[14] W. G. Unruh. Phys. Rev. Lett., 46:1351-1353, 1981.

[15] T. Jacobson. Phys. Rev. D, 44:1731-1739, 1991.

[16] W. G. Unruh. Phys. Rev. D, 51:2827-2838, 1995.

[17] R. Balbinot, A. Fabbri, S. Fagnocchi, and R. Parentani. Riv. Nuovo Cim. 28:1-55, 2005 (gr-qc/0601079).

[18] J. Martin and R. Brandenberger. Phys. Rev. D, 63:123501, 2001.

[19] J. Martin and R. Brandenberger. Phys. Rev. D, 68:063513, 2003.

[20] J. C. Niemeyer. Phys. Rev. D, 63:123502, 2001.

[21] J. C. Niemeyer and R. Parentani. Phys. Rev. D, 64:101301, 2001.

[22] C. Barrabès, V. Frolov, and R. Parentani. Phys. Rev. D, 62:044020, 2000.

[23] R. Parentani. Phys. Rev. D, 63:041503, 2001.

[24] R. Parentani. Int. J. Theor. Phys., 41:2175-2200, 2002.

[25] R. Parentani. Int. J. Mod. Phys., A17:2721-2726, 2002. 